nephron

Practice
Nephron 2016;134:191-194

DOI: $10.1159 / 000449235$
Received: May 25, 2016

Accepted after revision: July 13, 2016

Published online: August 30, 2016

\title{
Glomerular and Tubular Kidney Stress Test: New Tools for a Deeper Evaluation of Kidney Function
}

\author{
Claudio Ronco ${ }^{a, b}$ Lakhmir S. Chawla ${ }^{c, d}$ \\ ${ }^{a}$ Department of Nephrology, Dialysis and Transplantation, San Bortolo Hospital, and ${ }^{\mathrm{b}}$ International Renal

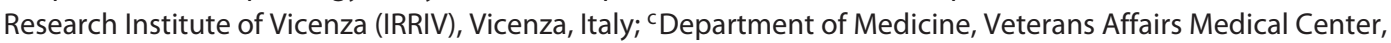 \\ and ${ }^{\mathrm{d}}$ Department of Medicine, George Washington University, Washington, D.C., USA
}

\section{Key Words}

Renal functional reserve - Kidney stress test .

Subclinical acute kidney injury - Acute kidney injury .

Chronic kidney disease - Glomerular filtration .

Tubular function · Furosemide stress test

\begin{abstract}
Kidney function has been traditionally identified with glomerular filtration rate (GFR). However, the kidney has multiple functions beyond filtration and a careful evaluation of its whole spectrum of activities should be carried out. Furthermore, the kidney presents a functional reserve capacity both at glomerular and tubular level. In presence of appropriate stimuli, patients with intact nephron mass can increase their GFR and tubular secretion. The difference between maximal capacity and baseline function represents the renal functional reserve (RFR). Glomerular and tubular kidney stress test may help to evaluate RFR and improve the clinicians' ability to better diagnose and prognosticate both acute and chronic kidney disease.

(c) 2016 S. Karger AG, Basel
\end{abstract}

\section{KARGER}

(c) 2016 S. Karger AG, Basel

E-Mail karger@karger.com

www.karger.com/nef

\section{Introduction}

While kidneys operate normally at baseline capacity, they can adapt to an increased demand or stress exploiting full functional capacity [1]. In presence of appropriate stimuli, patients with intact nephron mass can in fact increase glomerular filtration rate (GFR) and tubular secretion (TS) [2-4]. The difference between maximal functional capacity and baseline function represents the renal functional reserve (RFR) $[5,6]$. The ability to test this reserve may represent an excellent diagnostic possibility to unveil subclinical disease or silent loss of nephron mass $[7,8]$.

In the past, functional tests such as dilution or concentration capacity or urine acidification have been utilized to assess kidney function beyond baseline conditions.

Selected paper from a presentation at the 2016 AKI and CRRT UABUCSD O'Brien Center Symposium, San Diego, Calif., USA, February 16,2016 . This symposium was supported in part from a National Institutes of Health grant for the UAB-UCSD O'Brien Center for Acute Kidney Injury Research (P30 DK079337). 
The assessment of GFR and TS before and after stimuli may represent an interesting option to evaluate RFR [9, 10]. Glomerular and tubular kidney stress test (KST) may help to evaluate RFR and improve the clinicians' ability to better diagnose and prognosticate both acute and chronic kidney disease (CKD).

\section{RFR-Glomerular}

Serum creatinine (sCr) tends to remain normal even in presence of a solitary kidney when 1 of the 2 organs is removed [5]. In CKD sCr is still within normal range in the presence of a progressive nephron loss (up to 50\%). For this reason, $\mathrm{sCr}$ cannot be considered an accurate marker of renal function if GFR $>60 \mathrm{ml} / \mathrm{min} / 1.73 \mathrm{~m}^{2}$ [11]. Similarly, GFR estimation (eGFR) by creatinine-derived equations cannot be considered a sensitive index of renal function in the early phases of parenchymal damage or in moderate kidney disease [12]. In normal subjects, baseline GFR (bGFR) tends to change throughout the day depending on physiological requirements. GFR can also increase from baseline due to stimuli such as acute oral protein load or intravenous amino acid infusion [3-5]. This capacity to increase GFR in response to a stimulus is known as glomerular RFR (RFR-G). bGFR (unstressed), a surrogate of kidney function, averages in healthy subjects from 110 to $130 \mathrm{ml} / \mathrm{min} / 1.73 \mathrm{~m}^{2}$ in females and males, respectively. It changes with age, sex and body size, with considerable variation among individuals depending on diet and other situations [1]. Normal subjects display a significant capacity to increase GFR under physiological stimuli (e.g., pregnancy, solitary kidney) or pathological states (e.g., diabetes and hypertension) [13-16]. The difference between 'maximum' GFR and bGFR describes the RFR-G.

RFR-G is lower in the elderly and in the initial CKD stages although $\mathrm{sCr}$ is still normal [14]. Women at different stages of pregnancy display different bGFR due to progressive utilization of RFR-G. This explains why women with subclinical CKD often have higher rates of miscarriage [13].

Estimation of RFR-G can be made by short-term oral protein loading or by intravenous amino acid infusion. The increase in GFR in subjects with intact RFR-G varies between 30 and $70 \mathrm{ml} / \mathrm{min} / 1.73 \mathrm{~m}^{2}$. The mechanism of this response is likely to be linked to a release of nitric oxide and prostaglandins, resulting in vasodilation, increase of renal blood flow and GFR in the presence of a constant of filtration fraction [5]. This is the basis of a glomerular
KST that can be used to reveal subclinical kidney disease (fig. 1). When patients do not have obvious kidney disease, the loss of RFR-G is an indicator of silent loss of functioning nephron mass.

\section{Tubular Reserve}

The renal tubule portion of the nephron presents a variety of functions. Tubular reserve (TR) can be inferred by stressing the secretion capacity (TS) of the kidney tubule and it may be quite informative in different situations [9, 10, 17-20]. In most forms of kidney disease, the organs shrink and become more echogenic due to replacement of diseased tubules by matrix and fibrosis. Various studies have been conducted to determine the utility of testing TS capacity to predict outcomes $[9,10]$. TS of acid or sodium can be assessed by acid or salt loading, while tubular concentrating capacity can be assessed by water deprivation or exogenous administration of desmopressin acetate. TS can be assessed by measuring either creatinine or an exogenous drug (e.g., furosemide). Healthy patients are able to increase TS of creatinine (TScr), but CKD patients are not, probably because they are operating at near their maximum TScr. TScr has also been correlated with CKD progression although this concept remains a research tool and it has not yet been deployed into the clinics for CKD.

\section{Furosemide Stress Test}

Another possibility to evaluate TS capacity is to use intravenous furosemide $[9,10]$. Because furosemide is not filtered by the glomerulus and only eliminated by exclusive secretion in the proximal tubule, the measurement of amount of furosemide secreted in the urine after an intravenous bolus might allow the separation of urine output response from the proximal tubular function, resulting in an effective kidney tubular stress test (FST).

The FST has been prospectively assessed in a cohort of critically ill patients with acute kidney injury (AKI) displaying an excellent diagnostic performance $[9,10]$. A standard dose of intravenous furosemide was delivered to critically ill patients with KDIGO stage I or II AKI, and then urine output response was assessed. A 2-hour urine output $<100 \mathrm{ml} / \mathrm{h}$ in response to a furosemide challenge predicts progression to KDIGO stage III within 14 days with a receiver operator characteristic area under of the curve (AUC) of 0.87 (SE 0.05; fig. 1). At a cutoff of $200 \mathrm{ml}$ 


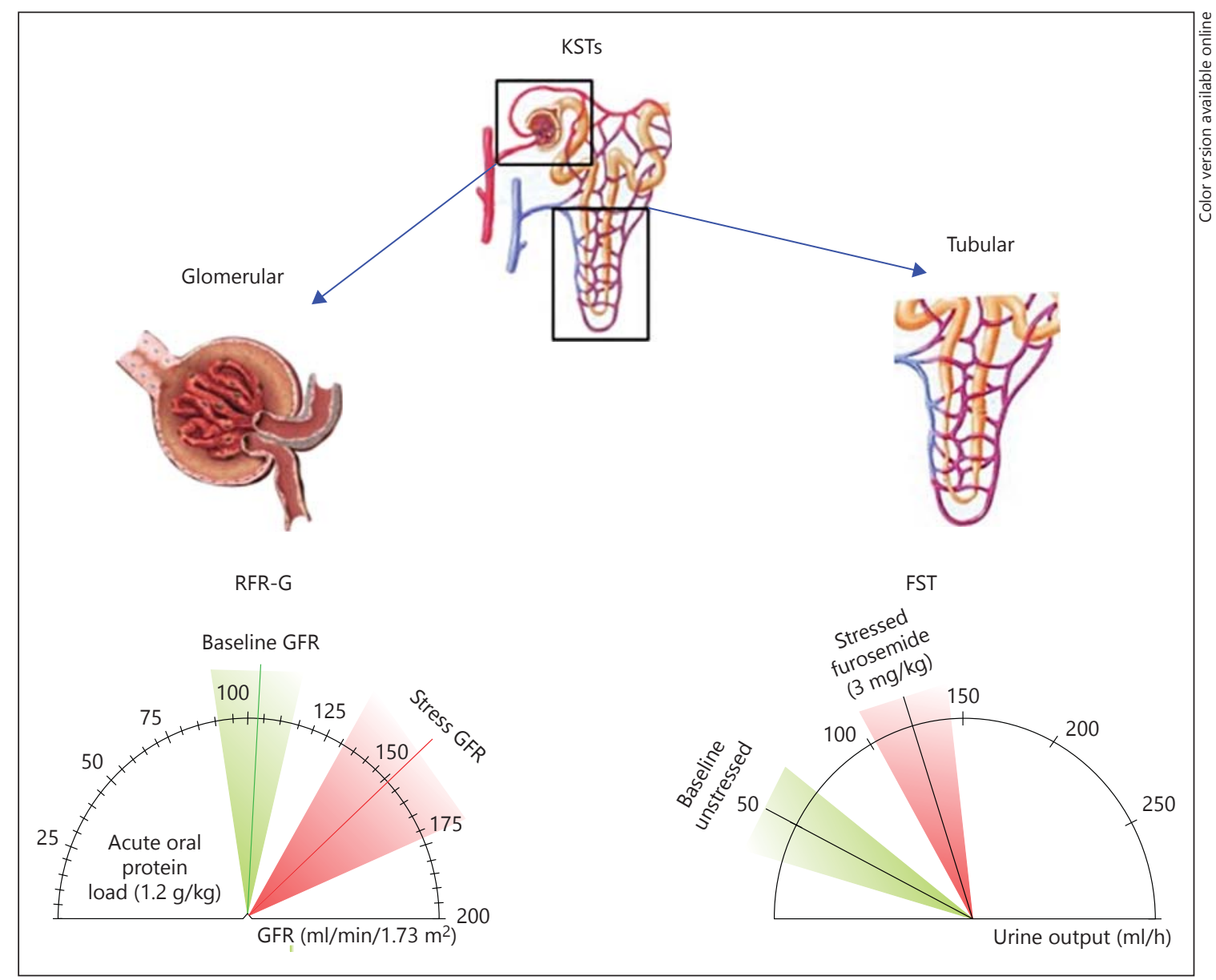

Fig. 1. Glomerular stress test (RFR-G): baseline creatinine clearance is measured with 2 one-hour periods before protein meal is administered. Twenty milliliter per kilogram oral hydration is performed before the test. Urine output is then replaced milliliter by milliliter. Protein meal ( $1.2 \mathrm{~g} / \mathrm{kg}$ of meat or powder protein) is administered in $30 \mathrm{~min}$. Bladder is then emptied and 3 one-hour period creatinine clearance measurements are performed. In the figure, we report the average values observed with marginal differences between males and females. The shaded areas represent 2
SDs. FST: the test is conducted by giving i.v. furosemide to patients with stage I or II AKI. The urine output should be replaced with isotonic fluid so that the FST is isovolemic. The dose is $1.0 \mathrm{mg} / \mathrm{kg}$ in patients who have not been exposed to a loop diuretic for 7 days, and the dose is $1.5 \mathrm{mg} / \mathrm{kg}$ for patients who have been given a loop diuretic within 7 days. Patients who are able to generate a urine output of $100 \mathrm{ml} / \mathrm{h}$ for $2 \mathrm{~h}$ have a better prognosis than those patients who are unable to achieve that threshold. at $2 \mathrm{~h}$, the sensitivity and specificity of the FST is 87.1 and $84.1 \%$, respectively. In order to assess the use of FST with AKI biomarkers, the FST was also tested amongst the patients with elevated levels of AKI biomarkers (i.e., TIMP2/IGFBP-7). In the subset of patients with elevated AKI biomarkers, the performance of the FST improved further $(\mathrm{AUC}=0.90)$. This is important as this is similar to the way in which stress testing is utilized in patients with cardiac disease - only patients with 'positive' troponin levels have subsequent stress testing. Similarly, when the FST is restricted to the group of patients with increased risk, the stress test is more informative. These data sug- gest that the combination of AKI biomarkers with TR assessment are informative and can be used at the bedside to assist clinicians in assessing the severity of AKI. FST does not just measure the tubule's secretion capacity, but it is actually an assessment of integrated renal function. In order for furosemide to increase urine output, furosemide must be actively secreted into the proximal lumen, and the functions of the thick ascending limb, luminal patency and collecting duct must all be intact.

Interest is growing on the potential of FST to predict the progression of CKD. The knowledge of which patients are likely to progress rapidly to ESRD is a critical 
clinical question that would help clinicians prepare patients for dialysis. Improved prognostic clarity might avoid the unnecessary placement of arteriovenous fistula and vascular access. These same techniques could also be applicable to patients with kidney transplants as a way to prognosticate delayed graft function and long-term allograft survival.

\section{Conclusions}

KST can be accomplished by assessing RFR-G and TR and both tests are safe, simple and relatively inexpensive. These tests have been shown to be informative both in AKI and CKD. RFR-G can be used to reveal the loss of nephron mass and unveil the risk for kidney disease. TR testing, although less developed, demonstrates excellent diagnostic performance and the capacity to endophenotype, prognosticate and better follow patients with kidney disease. The simple yet consistent rationale will definitely represent the base for future expansion of clinical and research application of the KSTs. In conjunction with new biomarkers and a better understanding of pathophysiological foundation of kidney disease, KST will hopefully help to make a step forward in diagnosis and care of kidney patients.

\section{Disclosure Statement}

The authors declare no conflict of interest for this manuscript. L.S.C. reports receiving consulting fees from Astute Medical, Baxter Medical and Nxstage Medical.

\section{References}

$>1$ Koopman MG, Koomen GC, Krediet RT, de Moor EA, Hoek FJ, Arisz L: Circadian rhythm of glomerular filtration rate in normal individuals. Clin Sci (Lond) 1989;77:105-111.

-2 Brenner BM, Meyer TW, Hostetter TH: Dietary protein intake and the progressive nature of kidney disease: the role of hemodynamically mediated glomerular injury in the pathogenesis of progressive glomerular sclerosis in aging, renal ablation, and intrinsic renal disease. N Engl J Med 1982;307:652-659.

$\checkmark 3$ Bosch JP, Saccaggi A, Lauer A, Ronco C, Belledonne M, Glabman S: Renal functional reserve in humans. Effect of protein intake on glomerular filtration rate. Am J Med 1983;75: 943-950.

4 Graf H, Stummvoll HK, Luger A, Prager R: Effect of amino acid infusion on glomerular filtration rate. N Engl J Med 1983;308:159160.

5 Sharma A, Mucino MJ, Ronco C: Renal functional reserve and renal recovery after acute kidney injury. Nephron Clin Pract 2014;127: 94-100.

6 Ronco C, Rosner MH: Acute kidney injury and residual renal function. Crit Care 2012; 16:144.

7 De Nicola L, Blantz RC, Gabbai FB: Renal functional reserve in treated and untreated hypertensive rats. Kidney Int 1991;40:406- 14 Barai S, Gambhir S, Prasad N, Sharma RK, 412.

$>8$ Ronco C, Kellum JA, Haase M: Subclinical AKI is still AKI. Crit Care 2012;16:313.

$\checkmark 9$ Chawla LS, Davison DL, Brasha-Mitchell E, Koyner JL, Arthur JM, Shaw AD, et al: Development and standardization of a furosemide stress test to predict the severity of acute kidney injury. Crit Care 2013;17:R207.

10 Koyner JL, Davison DL, Brasha-Mitchell E, Chalikonda DM, Arthur JM, Shaw AD, et al: Furosemide stress test and biomarkers for the prediction of AKI severity. J Am Soc Nephrol 2015;26:1747-1754.

-11 Delanaye P, Schaeffner E, Ebert N, Cavalier E, Mariat C, Krzesinski JM, et al: Normal reference values for glomerular filtration rate: what do we really know? Nephrol Dial Transplant 2012;27:2664-2672.

12 Levey AS, Bosch JP, Lewis JB, Greene T, Rogers N, Roth D: A more accurate method to estimate glomerular filtration rate from serum creatinine: a new prediction equation. Modification of diet in renal disease study group. Ann Intern Med 1999;130:461-470.

13 Ronco C, Brendolan A, Bragantini L, Chiaramonte S, Fabris A, Feriani M, et al: Renal functional reserve in pregnancy. Nephrol Dial Transplant 1988;3:157-161. Ora $\mathrm{M}$ : Functional renal reserve capacity in different stages of chronic kidney disease. Nephrology (Carlton) 2010;15:350-353.

15 Fliser D, Zeier M, Nowack R, Ritz E: Renal functional reserve in healthy elderly subjects. J Am Soc Nephrol 1993;3:1371-1377.

16 Pecly IM, Genelhu V, Francischetti EA: Renal functional reserve in obesity hypertension. Int J Clin Pract 2006;60:1198-1203.

17 Di Micco L, Quinn RR, Ronksley PE, Bellizzi V, Lewin AM, Cianciaruso B, et al: Urine creatinine excretion and clinical outcomes in CKD. Clin J Am Soc Nephrol 2013;8:18771883.

18 Herrera J, Rodríguez-Iturbe B: Stimulation of tubular secretion of creatinine in health and in conditions associated with reduced nephron mass. Evidence for a tubular functional reserve. Nephrol Dial Transplant 1998;13: 623-629.

19 Rodríguez-Iturbe B, Herrera J, Marín C, Mañalich R: Tubular stress test detects subclinical reduction in renal functioning mass. Kidney Int 2001;59:1094-1102.

20 van der Voort PH, Boerma EC, Pickkers P: The furosemide stress test to predict renal function after continuous renal replacement therapy. Crit Care 2014;18:429. 